\title{
Connections Between Layer 4B of Area 17 and the Thick Cytochrome Oxidase Stripes of Area 18 in the Squirrel Monkey
}

\author{
Margaret S. Livingstone and David H. Hubel \\ Department of Animal Neurobiology, Harvard Medical School, Boston, Massachusetts 02115
}

In area 18 of the primate visual cortex, staining for the mitochondrial enzyme cytochrome oxidase reveals 3 types of stripelike subdivisions running perpendicular to the $17 / 18$ border: thick, thin, and pale stripes. In a previous paper (Livingstone and Hubel, 1984), we described the anatomical connections with area 17 of 2 of these 3 subdivisions, but we did not have any conclusive information on the third subdivision, the thick stripes. Here we report that, in the squirrel monkey, the main input to the thick stripes from area 17 arises from layer $4 B$. Layer $4 B$ receives its input from the magnocellular division of the lateral geniculate body by way of layer $4 \mathrm{C} \alpha$; the thick stripes therefore probably belong to the magnocellular subdivision of the visual pathway.

Staining primate visual cortex for the mitochondrial enzyme cytochrome oxidase reveals regular patterns in both the primary visual cortical area (area 17) and the second visual cortical area (area 18) (Hendrickson et al., 1981; Horton and Hubel, 1981; Tootell and Silverman, 1981; Livingstone and Hubel, 1982, 1984; Humphrey and Hendrickson, 1983; Tootell et al., 1983; Hendrickson, 1985). The pattern in the upper layers of area 17 consists of small (100-150-mm-diameter) dark-staining blobs on a lighter-staining background. The pattern in area 18 is much coarser and consists of dark and light stripes, about $1 \mathrm{~mm}$ wide, running perpendicular to the $17 / 18$ border. The dark stripes seem to be of 2 alternating kinds, separated by pale stripes. In the squirrel monkey, one usually can distinguish the 2 different types of dark stripes in the cytochrome oxidase pattern because one type is usually thicker than the other (see, for example, Figs. $1 b, 2 b, 3 d)$, but in the macaque it is more difficult to distinguish 2 types of dark stripes from cytochrome oxidase staining alone. Evidence from anatomical studies (Livingstone and Hubel, 1984; DeYoe and Van Essen, 1985; Shipp and Zeki, 1985), physiological studies (Hubel and Livingstone, 1985), and deoxyglucose uptake studies (Tootell et al., 1983) nevertheless suggests that macaques also have 2 alternating types of stripes in area 18, and that the 2 types are homologous to the thick and thin stripes in the squirrel monkey. The significance of the dark and light

\footnotetext{
Received Dec. 2, 1985; revised Apr. 13, 1987; accepted Apr. 13, 1987.

The histology was done by Debra Broihier and Janet Robbins, the photography by Richard McDonald. The manuscript benefited from comments from David Cardozo, Roger Tootell, and anonymous referees. This work was supported by NIH Grants EY00605 and KO4 NS00972, a Presidential Young Investigator Award from the NSF, Office of Naval Research Grant N00014-85-K-0447, a Young Investigator Award from the Office of Naval Research, and funds from the Monsanto Company, F. I. Dupont de Nemours and Co., and the Fairchild Laboratories.

Correspondence should be addressed to Margaret $\mathrm{S}$. Livingstone at the above address.

Copyright (C) 1987 Society for Neuroscience $0270-6474 / 87 / 113371-07 \$ 02.00 / 0$
}

staining for the enzyme cytochrome oxidase is unknown (except that it probably reflects differences in chronic levels of neuronal activity), but the pattern of staining is nonetheless very useful as an anatomical marker because it correlates with physiologically and other anatomically distinct subdivisions within the visual system.

In a previous paper we showed that the blobs of area 17 are reciprocally connected to the thin stripes of area 18 , and the interblob regions of 17 to the pale stripes of 18 (Livingstone and Hubel, 1984). In that study we were unable to obtain any information on the connections of the thick stripes with area 17. Of the 35 horseradish-peroxidase injections that we made into squirrel monkey area 18 , the injections that landed in either thin stripes or pale stripes usually produced strong and patchy labeling in area 17, whereas injections into thick stripes produced much weaker, diffuse labeling in 17 , which did not show any clear relationship to the cytochrome oxidase pattern. We concluded, tentatively, that both blob and non-blob regions of area 17 project weakly, if at all, to the thick stripes.

One hint as to the significance of the specificity of these interconnections comes from the physiology. In area 17, the darkstaining blobs and the lighter-staining interblob regions differ strikingly in their physiological properties: cells in the interblobs respond best to lines or edges of particular orientations, and only a minority are selective for the color of visual stimuli, whereas cells in the blobs are not orientation-selective, and many are color-coded. In subsequent single-unit recording experiments in area 18 we have found that the cells in each of the different types of stripes likewise have distinctive physiological properties that are consistent with their specific connections with area 17 (Hubel and Livingstone, 1985, 1987). Cells in the thin stripes, like cells in the blobs of 17 to which they are connected, are not orientation-selective, and many of them are color-coded. Cells in the pale stripes, like cells in the interblobs of 17 , are orientation-selective but not color-selective, suggesting that this system is concerned primarily with information about shape. Visual information about form thus seems to become segregated from information about color. Although in their physiological properties, cells in the thin stripes and cells in the pale stripes show similarities to their antecedent cells in area 17 (in the blobs and the interblobs), they also show consistent differences. In the blob/thin-stripe system, the information in 18 becomes more generalized as to spatial localization, in that the size of region of the visual field within which a cell responds to stimuli increases, but the optimum stimulus size does not change. We term this operation "complexification," by analogy to the difference between simple and complex cells (Hubel and Livingstone, 1985). In the interblob/pale-stripe 

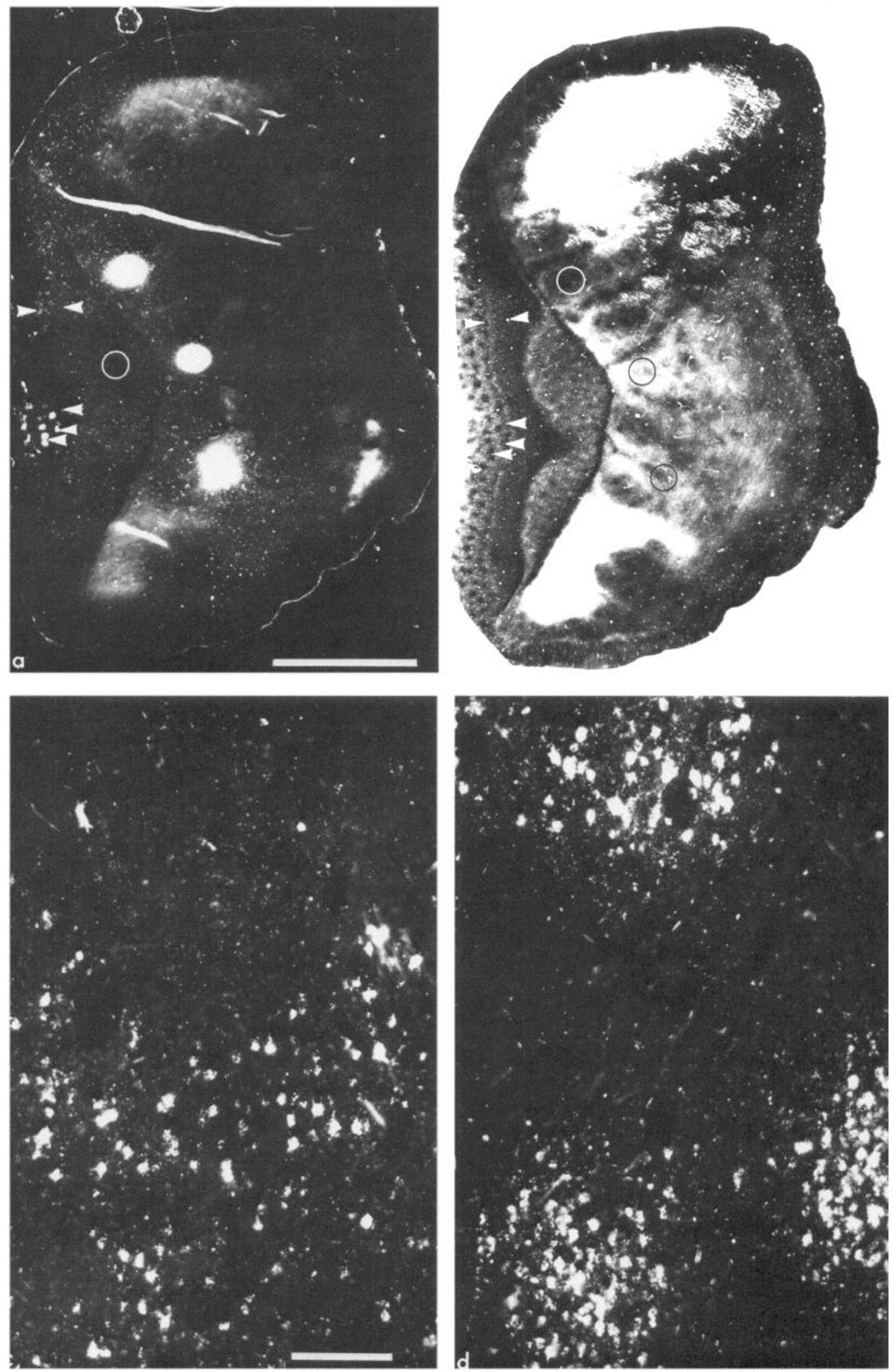

Figure 1. $a$ and $b$, Low-power views of 2 adjacent sections from the occipital lobe of a squirrel monkey, in which 3 horseradish-peroxidase injections were made into area 18. Arrowheads indicate the same blood vessels in the 2 sections. Scale bar, $5 \mathrm{~mm}$. $a$, Section treated with tetramethylbenzidine and photographed through perpendicularly aligned polarizers, showing 3 injections in area 18, as well as label transported to area 17 for 2 of the injections (the top and bottom injections). The region in 17 to which label was transported from the middle injection is shown by the circle, but because of the curvature of the cortex, the label was present only in more superficial sections, which passed through the upper layers in that region. $b$, Next-deeper section, stained for cytochrome oxidase. Injection sites are indicated by circles. The top injection was in a thick stripe, and the label in area 17 was found exclusively in layer $4 \mathrm{~B}$, as can be seen by comparing the positions of the 2 blood vessels marked by arrowheads to the location of the labeled cells in $a$ to the same region in the adjacent section $(b)$. The bottom injection, in a thin stripe, produced 
system, a higher proportion of cells in 18 is end-stopped, responding to short, but not long, lines or edges. Last, we found that units in the thick stripes were often precisely tuned for binocular disparity, suggesting the existence of a third separate pathway, one specifically involved in stereoscopic depth perception. Although stereotuned cells have been observed in area 17 (Poggio and Fischer, 1977), they have so far not been assigned to any anatomical subdivision within 17 .

Because of these distinctive physiological properties of the cells in the thick stripes, we redoubled our efforts to learn which cells in area 17 , if any, project to them by making 36 more wheat germ agglutinin-conjugated horseradish-peroxidase injections blindly into area 18 of 9 squirrel monkeys and looking for transported label in area 17 . We used only squirrel monkeys and no macaques in this study, even though much of our physiology has been done in macaques, because differentiation between thick and thin stripes is much easier in squirrel monkeys. As mentioned above, the physiological studies described in the following paper (Hubel and Livingstone, 1987), as well as anatomical and deoxyglucose studies, suggest that macaques also have 2 alternating kinds of cytochrome-dark stripes homologous to the thick and thin stripes in squirrel monkeys, even though a distinction between them is not obvious from the cytochrome oxidase pattern alone.

\section{Materials and Methods}

The methods of anesthesia, tracer injection, perfusion, and staining were exactly as described in part 4 of Livingstone and Hubel (1984). Animals were initially anesthetized with ketamine and were kept anesthetized throughout the experiment with $1.5 \%$ halothane in air. Burr holes about $2 \mathrm{~mm}$ in diameter were drilled in the skull and small holes made in the dura. Horseradish peroxidase, wheat-germ conjugated (Sigma), was dissolved in $0.1 \mathrm{M}$ Tris buffer, $\mathrm{pH} 8.6$, at a concentration of $5 \% \mathrm{wt} / \mathrm{vol}$ and injected iontophoretically through glass micropipettes (tip diameter, $10-20 \mu \mathrm{m}$ ) using a $1 \mu \mathrm{A}$ squarewave current, electrode-positive, $5 \mathrm{sec}$ on and $10 \mathrm{sec}$ off, for $5 \mathrm{~min}$. Survival times were approximately $72 \mathrm{hr}$, after which the animals were anesthetized with sodium thiopental and perfused transcardially with 1 liter of physiological saline, followed by 1 liter of fixative $(0.75 \%$ paraformaldehyde, $2.25 \%$ glutaraldehyde, $0.9 \%$ $\mathrm{NaCl}, 0.1 \mathrm{~m}$ sodium phosphate buffer, $\mathrm{pH} 7.4)$. Sections $30-60 \mu \mathrm{m}$ thick were cut on a freezing microtome, and alternate sections were treated by the method of Wong-Riley (1979b) to detect cytochrome oxidase or by the method of Mesulam (1982) to detect the horseradish peroxidase.

\section{Results}

Many of the tracer injections fell into thin stripes or pale stripes, and, in confirmation of our earlier results, we again found that whenever the injection site was in a thin stripe in area 18, the label in 17 was restricted to blobs, and that whenever the injection was in a pale stripe, the label in 17 was in interblob regions.

We ultimately succeeded in making 2 injections that were clearly in thick stripes and resulted in filled cell bodies in area 17. In both of these experiments the filled cells in area 17 were confined to layer 4B. In the experiment shown in Figure 1, we made 3 horseradish-peroxidase injections into area 18 on the right side. Figure $1 a$ shows a section treated to show horseradish peroxidase, and Figure $1 b$ shows the next-deeper section, stained for cytochrome oxidase. The injections can be seen in Figure $1 a$ as 3 bright spots $1-1.5 \mathrm{~mm}$ wide, and their corresponding positions in Figure $1 b$ are indicated by circles.

The most posterior of the 3 injections (the bottom injection in Fig. 1a) was centered on a thin stripe but also appeared to extend into the adjacent pale stripes. It produced clumps of intense labeling in area 17 , seen at the very left of Figure $1 a$. By comparing the positions of the 3 blood vessels, marked in Figure 1, $a$ and $b$, with arrowheads, the labeled cells can be seen to lie in layers 2 and 3 , exclusively in blobs. That this injection produced labeling in blob cells but not in non-blob cells suggests that only the center of the injection produced labeling in area 17. The photograph in Figure $1 d$ shows 3 of the labeled patches from the region labeled by the bottom injection. We chose to photograph these patches, which were at the edge of the labeled region and were among the least densely labeled patches, in order to be able to show individual filled cells.

The top (anterior) injection was entirely within a thick stripe. The corresponding horseradish-peroxidase label in area 17 was much weaker than the label from the bottom injection, even though the 2 injections were about the same size. The label can be seen, faintly, between the 2 arrowheads just to the left of the injection site in Figure $1 a$, closer to the left-hand arrowhead. The arrowhead tips mark blood vessels, and by using these as landmarks and comparing the sections in Figure $1, a$ and $b$, one can see that the labeled cells lie in the layer that stains very lightly for cytochrome oxidase, i.e., layer 4B. Because the plane of section was not tangential to the surface in the region of the labeling, we could not tell whether the location of the filled cells bore any relationship to the overlying blob pattern. Though the section in Figure la shows the maximum region of labeling in 17 for the top injection, it is only one of several sections in which the transported label from the bottom injection was much brighter. A higher-power view of the label transported from the top injection is shown in Figure $1 c$. The labeled cell bodies are all located in layer 4B, but in layer 3 there is some diffuse, probably anterograde, label superficial to the filled cell bodies.

The middle injection was centered on a thick stripe but extended into the adjacent pale stripes. (We assume this is a thick stripe because of the clear alternating pattern of stripes anterior and posterior to it, but this particular stripe is unusually pale; we have noticed in many squirrel monkeys that the central, foveal, thick stripe is often anomalous.) The region of area 17 labeled as a result of this injection is indicated in Figure $1 a$ by the white circle. Because of the curvature of the cortex, the label is present only in more superficial sections. The labeled cells were in layers 2 and 3 , exclusively in interblobs, and also in layer 4B. This pattern of labeling in 17 would be consistent with

much brighter labeling, which can be seen to the left of the 3 arrowheads; by comparing the positions of the arrowheads in $a$ and $b$, one can see that the labeled cells are all in layers 2 and 3 and are exclusively in blobs. [The blood vessel marked by the bottom (most posterior) arrowhead lies at the base of 2 labeled patches in $a$ and at the base of a pair of blobs in $b$; the arrowhead just above and to the right of the bottom arrowhead marks a blood vessel at the base of a single patch of label in $a$ and at the base of a blob in $c$; the upper arrowhead marks a vessel about $100 \mathrm{~mm}$ to the right of a patch of label in $a$ and a blob in $b$.] $c$ and $d$, High-power views of the section shown in $a$. The orientation is orthogonal to that in $a$, so up is towards the pial surface. Scale bar, $100 \mu \mathrm{m} . c$, Cells in area 17 labeled by the top, thick-stripe, injection. The upper third of the photograph is layer 3 and the lower two-thirds layer 4B. Labeled cell bodies can be seen in 4B and diffuse labeling in layer 3. $d$, Cells labeled by the bottom, thin-stripe, injection. 

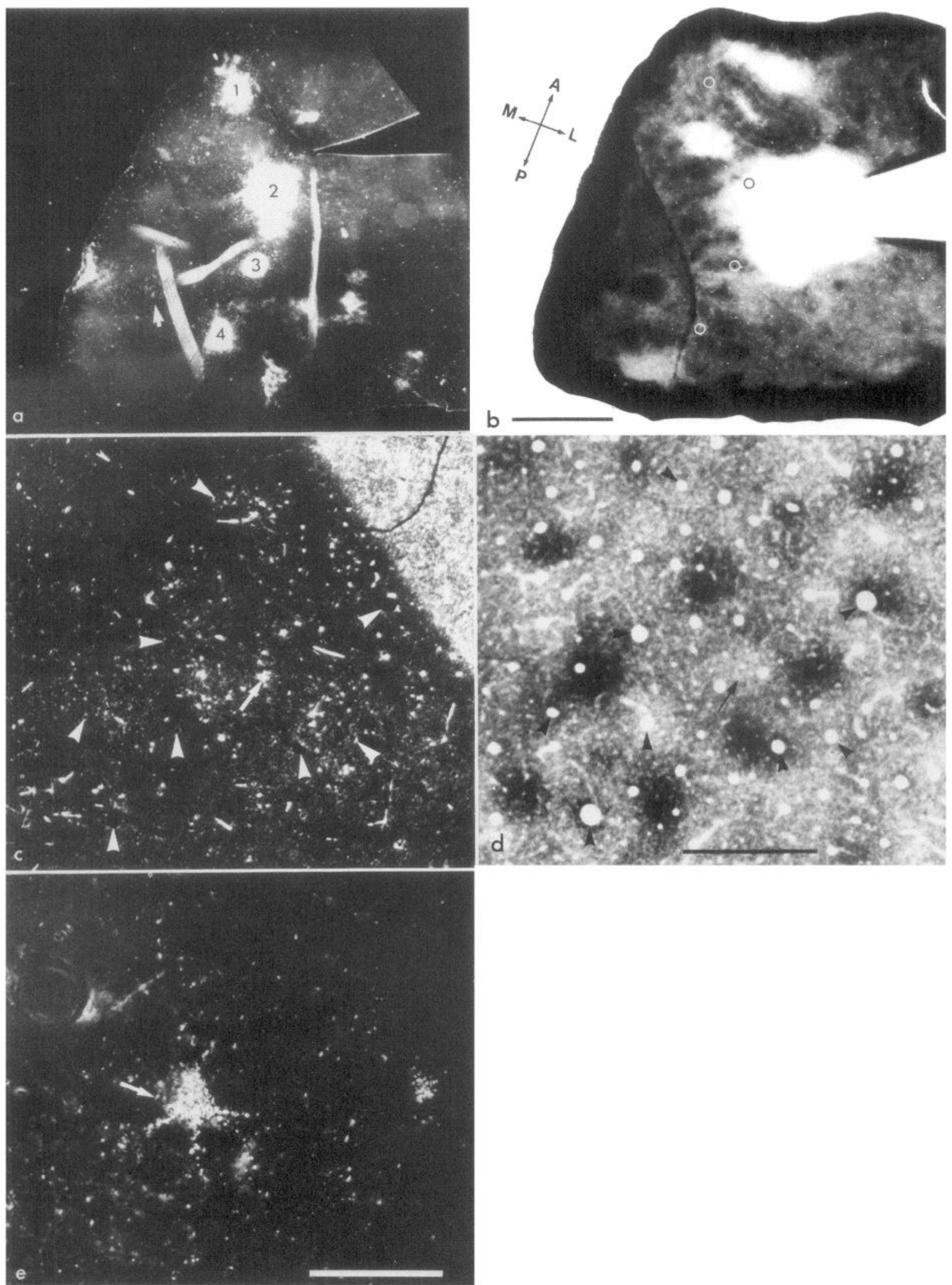

Figure 2. $a$ and $b$, Low-power views of 2 sections from the occipital lobe of a squirrel monkey with 4 horseradish-peroxidase injection sites in area 18. Scale bar, $5 \mathrm{~mm}$. $a$. Tetramethylbenzidine-treated section photographed through crossed polarizers. (The 4 thick bright lines are folds in the section.) Several patches of label to the right of the 4 injections probably represent connections from area 18 to the further prestriate areas, since the label is anterior to the stripes, which probably define the boundaries of area 18 . We cannot tell which injections produced which prestriate labeling, but it is worth noting how patchy these connections are. $b$, Section $300 \mu \mathrm{m}$ deeper than $a$, stained for cytochrome oxidase. Injection sites are indicated by circles. The cytochrome oxidase pattern was unclear in the region of the top injection; the second injection was large and covered 
our other results, since the injection included both pale stripes and a thick stripe.

In the experiment shown in Figure 2, we made 4 injections into area 18 on the right side. The section in Figure $2 a$, treated to show horseradish peroxidase, shows the injection sites, whose center locations are indicated by small circles in the cytochrome oxidase-stained section in Figure $2 b$. (The section shown in Fig. $2 b$ is $300 \mathrm{~mm}$ deeper than the one shown in Fig. $2 a$ because the stripe pattern in area 18 was clearer in the deeper layers.)

The cytochrome oxidase pattern in the region of the top injection was too unclear for us to tell which type of stripe it was in. The second injection was large and was centered at the border between a thin stripe and an interstripe. This injection produced the labeling at the far left edge of the section in Figure $2 a$; the label was heavy and extended over several sections. The labeled cells were in layers 2 and 3, both in blobs and interblobs, as would be expected from an injection site straddling a thin stripe and a pale stripe.

The third injection was in a thick stripe. Cells in area 17 labeled from this injection are located just above the arrow in Figure $2 a$. This was the only section with labeled cells in the part of 17 corresponding to this injection site, and these cells were located in layer 4B. Figure $2 c$ is a higher-power view of this region, showing the patches of labeled cells, and Figure $2 d$ is the same region in a more superficial, cytochrome oxidasestained section. The same blood vessels are marked by arrowheads in the 2 sections. Comparing Figure 2, $c$ and $d$, we can see that most of the labeled cells in this experiment lay under interblobs, but many labeled cells clearly lay outside blobs; thus, in this experiment and others, there did not appear to be any clear or consistent correspondence of the filled cells with the overlying blob pattern. Figure $2 e$ shows a higher-power view of the cell indicated by the arrow in Figure $2 c$, to prove to the anatomists that we know a cell when we see one.

The weakness of the label from thick stripes in these 2 experiments, compared to the projection from layer 3 to the thin stripes or the pale stripes, made us realize that we could easily have overlooked similar results in previous experiments, so we went back and reexamined 4 thick-stripe injections from our earlier experiments, and we did find faint labeling, again in layer $4 \mathrm{~B}$ exclusively, and again much weaker than the label from the thin stripe or interstripe injections.

In earlier studies, injections large enough to probably include more than one kind of stripe produced retrogradely labeled cells in area 17 in layers 3, 4B, 5, and 6 (Rockland and Pandya, 1979; Lund et al., 1981; Tigges et al., 1981; Kennedy and Bullier, 1985). Since, in order to label single stripes, we used much smaller injections, any projections weaker than that from $4 \mathrm{~B}$ would probably have been missed. Thus, though we did not see labcled cells in arca 17 from the thick-stripc injections anywhere other than in layer $4 \mathrm{~B}$, we cannot rule out additional weaker projections from layers 3,5 , or 6 .

\section{Injections in area 17}

The area 18 injections into thick stripes resulted in much less labeling in area 17 than did injections of similar size in thin stripes or interstripes. This suggests that the projection from 4B to the thick stripes is weaker than the projections from layers 2 and 3 to the thin stripes and the interstripes. Consistent with this, earlier studies with much larger horseradish-peroxidase injections into area 18 had shown that the great majority of back-labeled cells in area 17 were in layer 3, with many fewer cell bodies in layer 4B (Rockland and Pandya, 1979; Lund et al., 1981; Tigges et al., 1981; Kennedy and Bullier, 1985). Several earlier studies in various primates had shown that large injections of tracer into area 17 could result in periodic light and dark labeling in area 18 (Tigges et al., 1973; Martinez-Millán and Holländer, 1975; Rockland and Pandya, 1979; Wong-Riley, 1979a; Lund et al., 1981), and we naturally suspected that the lighter regions would correspond to the thick stripes. To see whether the projection from area 17 to the thick stripes is indeed weaker than the projection to the thin stripes and interstripes, we made large horseradish-peroxidase injections into area 17 of 2 squirrel monkeys and compared the anterograde transport to layer 4 of area 18 with the cytochrome oxidase pattern.

Figure 3 shows both hemispheres from one squirrel monkey in which we made one large horseradish-peroxidase injection into area 17 on each side. The injection in the left hemisphere can be seen at the upper right edge of the section in Figure $3 a$. The plane of section in Figure $3 c$ did not pass through the injection site on that side, but the injection was about the same size as the one on the other side. Both horseradish-peroxidase sections (Fig. 3, $a$ and $c$ ) show the transported label in layer 4 of area 18, the layer to which 17 projects (Spatz et al., 1970; Martinez-Millán and Holländer, 1975; Wong-Riley, 1976; Rockland and Pandya, 1979). In both hemispheres the label in area 18 is not uniform, but waxes and wanes. When the pattern is compared to the cytochrome oxidase pattern, using the blood vessels marked by arrowheads, the regions with the lightest labeling correspond to the thick stripes.

\section{Discussion}

We conclude that the main input from area 17 to the thick stripes in area 18 is from layer $4 \mathrm{~B}$, and that this input is probably much weaker than the inputs from layers 2 and 3 to the thin stripes and the interstripes. The relative weakness of the projection from area 17 to the thick stripes might seem inconsistent with the large fraction of 18 that they occupy, and with the relatively high level of activity suggested by the dark cytochrome oxidase staining. Possibly much of the activity of these stripes

both a thin stripe and an adjacent pale stripe (seen better in more superficial sections); the third injection was in a thick stripe; the bottom injection straddled the $17 / 18$ border. In $a$, the label transported from the third, thick-stripe, injection is located just above the small arrow, and just to the left of the fold. This was the only section in which we could find any labeling from the thick-stripe injection. The label transported to area 17 from the top injection was deep in the calcarine fissure, and is not present in this section. The labeling at the left edge of the section is transported from the second injection. This injection resulted in bright labeling in several consecutive sections in layers 2 and 3 , in both blobs and non-blobs, as would be expected from an injection that included both a thin stripe and a pale stripe in area 18 . The label in area 17 near the bottom injection could not be attributed to transport from area 18 , because part of the injection itself extended into area 17 , and intrinsic connections in area 17 would have contributed to the pattern of labeling. $c$ and $d$, Higher-power views of the region labeled by the thick-stripe injection. The same blood vessels are marked with arrowheads in the 2 sections so that they can be compared. Scale bar, $0.5 \mathrm{~mm}$. $c$, Horseradish-peroxidase section showing filled cells. $d$. Same region as $c, 150 \mu \mathrm{m}$ more superficial in order to show overlying blob pattern. The locations of the labeled cells and the blob pattern show little correspondence. $e$, Higher-power view of the large cell indicated by the arrow in $c$. Scale bar, $50 \mu \mathrm{m}$. 

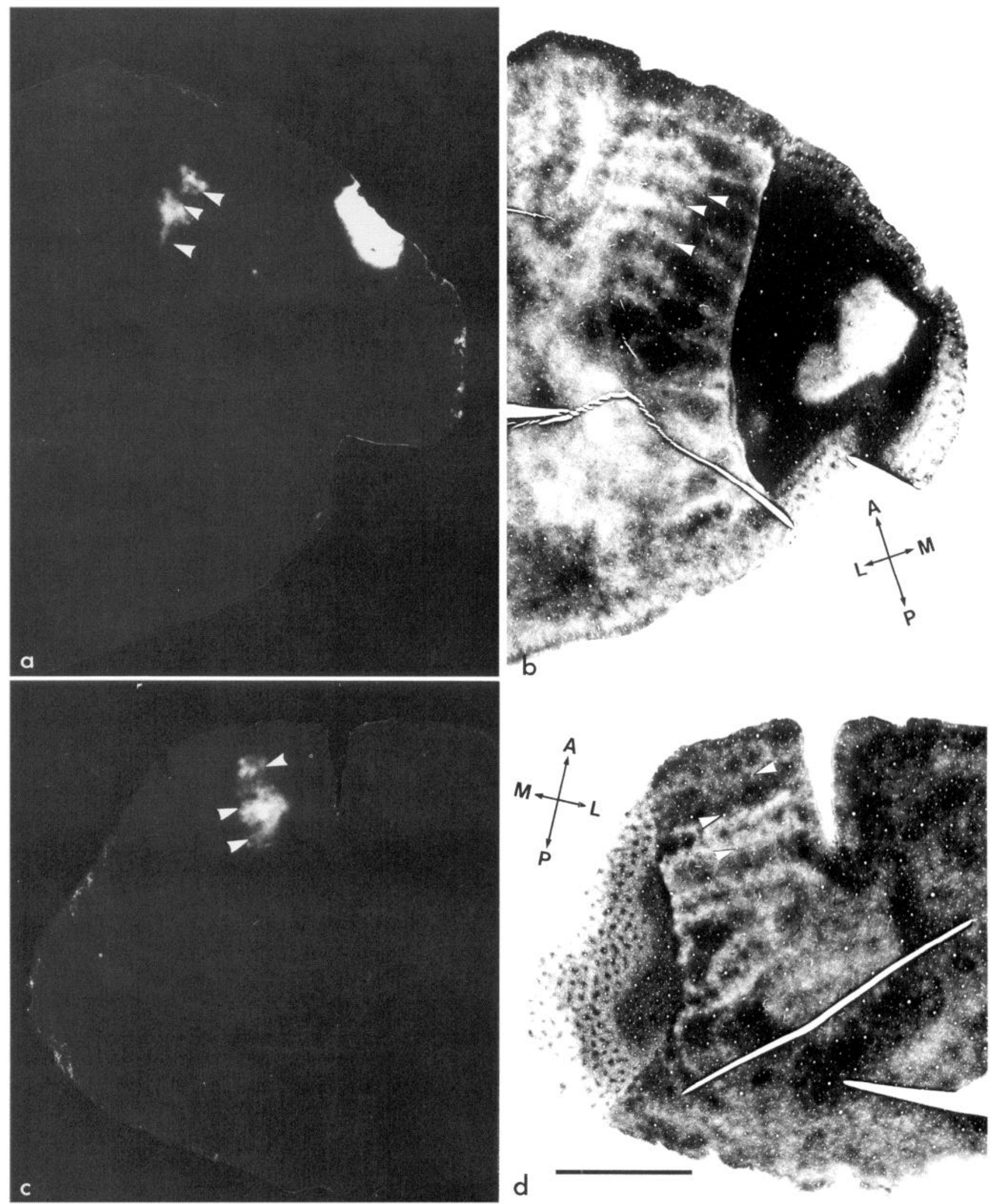

Figure 3. Low-power views of sections from the occipital lobes of both emispheres of a squirrel monkey with one large horseradish-peroxidase injection in area 17 on each side, showing the transported label in area 18. $a$ and $b$, From the left hemisphere; $c$ and $d$, from the right. The injection on the right side is visible as the bright white patch at the upper right edge of the section in $a$, but the plane of section in $c$ and $d$ did not pass through the injection site on the other side. Scale bar, $5 \mathrm{~mm} . a$ and $c$, Tetramethylbenzidine-treated sections photographed through crossed polarizers. The part of each section with the transported label is in layer 4 of area 18 , so the labeling should represent anterograde transport from area 17 . $b$ and $d$, Adjacent cytochrome oxidase-stained sections. When corresponding regions of sections $a$ and $b$ or $c$ and $d$ are compared by lining up the radially running blood vessels (arrowheads), the lightest labeling in area 18 is seen to lie in thick stripes. 
is driven by inputs from the pulvinar, from which they receive strong projections (Livingstone and Hubel, 1982), or from other cortical regions.

Layer 4B of primate area 17 receives its main input from the magnocellular geniculate layers via layer $4 \mathrm{C} \alpha$, whereas layers 2 and 3 receive their main input from the parvocellular layers via layer $4 \mathrm{C} \beta$ (Hubel and Wiesel, 1972; Lund, 1973). In the accompanying paper on the properties of the different subdivisions of area 18 (Hubel and Livingstone, 1987), we describe our finding that the thick stripes seem particularly involved with information about stereoscopic depth perception, and in the accompanying psychophysical paper (Livingstone and Hubel, 1987) we discuss the evidence that led us to suspect that the stereoscopic system might receive its main input from the magnocellular pathway. It was, in fact, this suspicion that led us, in the present study, to reinspect layer 4B carefully enough to find the cell bodies that better anatomists might have seen immediately. From the results of DeYoe and Van Essen $(1984,1985)$ and Shipp and Zeki (1985), it appears likely that the thick stripes, but not the thin stripes or the interstripes, are reciprocally interconnected with the prestriate area MT (or STS in the macaque), which is also directly and reciprocally connected with layer 4B of 17 (Zeki, 1969; Lund et al., 1975; Spatz, 1975; Ungerleider and Mishkin, 1979; Tigges et al., 1981; Maunsell and Van Essen, 1983). Thus, within the visual pathway, the components of the magnocellular system are interconnected and apparently strikingly segregated from the parvocellular pathway. As we describe in the following papers, the thick stripes are particularly involved with processing information about stereopsis and also, possibly, movement, and the possibility that the magnocellular, but not the parvocellular, geniculate system furnishes a major input to the thick stripes is not inconsistent with the psychophysics of stereopsis and movement.

\section{References}

DeYoe, E. A., and D. C. Van Essen (1984) Ncurons projecting to MT and $\mathrm{V} 4$ from macaque $\mathrm{V} 2$ are segregated into discrete stripe-like patches. Soc. Neurosci. Abstr. 10:934.

DeYoe, E. A., and D. C. Van Essen (1985) Segregation of efferent connections and receptive field properties in visual area $\mathrm{V} 2$ of the macaque. Nature 317: 58-61.

Hendrickson, A. E. (1985) Dots, stripes and columns in monkey visual cortex. Trends Neurosci. 8: 406-410.

Hendrickson, A. E., S. P. Hunt, and J.-Y. Wu (1981) Immunocytochemical localization of glutamic acid decarboxylase in monkey striate cortex. Nature 292: 605-607.

Horton, J. C., and D. H. Hubel (1981) A regular patchy distribution of cytochrome-oxidase staining in primary visual cortex of the macaque monkey. Nature 292: 762-764.

Hubel, D. H., and M. S. Livingstonc (1985) Complex-unoriented cells in a subregion of primate area 18 . Nature 315: 325-327.

Hubel, D. H., and M. S. Livingstone (1987) Segregation of form, color, and stereopsis in primate area 18. J. Neurosci. 7: 3378-3415.

Hubel, D. H., and T. N. Wiesel (1972) Laminar and columnar distribution of geniculo-cortical fibers in the macaque monkey. J. Comp. Neurol. 146: 421-450.

Humphrey, A. L., and A. E. Hendrickson (1983) Background and stimulus-induced patterns of high metabolic activity in the visual cortex (area 17) of the squirrel and macaque monkey. J. Neurosci. 3: 345-358.

Kennedy, H., and J. Bullier (1985) A double-labeling investigation of the afferent connectivity to cortical areas V1 and V2 of the macaque monkey. J. Neurosci. 5: 2815-2830.

Livingstone, M. S., and D. H. Hubel (1982) Thalamic inputs to cy- tochrome oxidase-rich regions in monkey visual cortex. Proc. Natl. Acad. Sci. USA 79: 6098-6101.

Livingstone, M. S., and D. H. Hubel (1984) Anatomy and physiology of a color system in the primate visual cortex. J. Neurosci. 4: 309356.

Livingstone, M. S., and D. H. Hubel (1987) Psychophysical evidence for separate channels for the perception of form, color, movement, and depth. J. Neurosci. 7: 3416-3468.

Lund, J. S. (1973) Organization of neurons in the visual cortex, area 17, of the monkey (Macaca mulatta). J. Comp. Neurol. 147: 455496.

Lund, J. S., R. D. Lund, A. E. Hendrickson, A. H. Bunt, and A. F. Fuchs (1975) The origin of efferent pathways from the primary visual cortex, area 17, of the macaque monkey as shown by retrograde transport of horseradish peroxidase. J. Comp. Neurol. 164: 287-304.

Lund, J. S., A. E. Hendrickson, M. P. Ogren, and E. A. Tobin (1981) Anatomical organization of primate visual cortex area VII. J. Comp. Neurol. 202: 19-45.

Martinez-Millán, L., and H. Holländer (1975) Cortico-cortical projections from striate cortex of the squirrel monkey (Saimiri sciureus). An autoradiographic study. Brain Res. 83: 405-417.

Maunsell, J. H. R., and D. C. Van Essen (1983) The connections of the middle temporal visual area (MT) and their relationship to a cortical hierarchy in the macaque monkey. J. Neurosci. 3: 2563-2586.

Mesulam, M.-M. (1982) Principles of horseradish peroxidase neurohistochemistry and their applications for tracing neural pathways: Axonal transport, enzyme histochemistry and light microscopic analysis. In Tracing Neural Connections with Horseradish Peroxidase, M.M. Mesulam, ed., pp. 1-151, Wiley, New York.

Poggio, G. G., and B. Fischer (1977) Binocular interaction and depth sensitivity in striate and prestriate cortex of behaving rhesus monkey. J. Neurophysiol. 40: 1392-1405.

Rockland, K. S., and D. N. Pandya (1979) Laminar origins and terminations of cortical connections of the occipital lobe in the rhesus monkey. Brain Res. 179: 3-20.

Shipp, S., and S. Zeki (1985) Segregation of pathways leading from area V2 to areas V4 and V 5 of macaque monkey visual cortex. Nature 315: 322-325.

Spatz, W. B. (1975) An efferent connection of the solitary cells of Meynert. A study with horseradish peroxidase in the marmoset $\mathrm{Cal}$ lithrix. Brain Res. 92: 450-455.

Spatz, W. B., J. Tigges, and M. Tigges (1970) Subcortical projections, cortical associations, and some intrinsic interlaminar connections of the striate cortex in the squirrel monkey (Saimiri). J. Comp. Neurol. 140: $155-174$.

Tigges, J., M. Tigges, and C. S. Kalaha (1973) Efferent connections of area 17 in Galago. Am. J. Phys. Anthropol. 38: 393-398.

Tigges, J., M. Tigges, S. Anschel, N. A. Cross, W. D. Ledbetter, and R. L. McBride (1981) Areal and laminar distribution of neurons interconnecting the central visual cortical areas 17,18,19, and MT in squirrel monkey (Saimiri). J. Comp. Neurol. 202: 539-560.

Tootell, R. B. H., and M. Silverman (1981) A comparison of cytochrome oxidase and deoxyglucose patterns in macaque visual cortex. Soc. Neurosci. Abstr. 7: 356.

Tootell, R. B. H., M. S. Silverman, R. L. De Valois, and G. H. Jacobs (1983) Functional organization of the second cortical visual area in primates. Science 220:737-739.

Ungerleider, L. G., and M. Mishkin (1979) The striate projection zone in the superior temporal sulcus of Macaca mulatta: Location and topographic organization. J. Comp. Neurol. 188: 347-366.

Wong-Riley, M. T. T. (1976) Autoradiographic studies of subcortical and cortical projections from striate and extrastriate cortices of squirrel and macaque monkeys. Anat. Rec. 184: 566.

Wong-Riley, M. T. T. (1979a) Columnar cortico-cortical interconnections within the visual system of the squirrel and macaque monkeys. Brain Res. 162: 201-217.

Wong-Riley, M. T. T. (1979b) Changes in the visual system of monocularly sutured or enucleated cats demonstrable with cytochrome oxidase histochemistry. Brain Res. 171: 11-28.

Zeki, S. (1969) Representation of central visual fields in prestriate cortex of monkey. Brain Res. 14: 271-291. 